\title{
FLUCTUACIÓN POBLACIONAL DE Tetranychus sp. EN Ipomoea purpurea (L.) Roth. ("CAMPANILLA") Y ESTABLECIMIENTO DE Euseius stipulatus (Athias-Henriot) EN MIRAFLORES / LIMA / PERÚ
}

\section{POPULATION FLUCTUATION OF Tetranychus sp. IN Ipomoea purpurea (L.) Roth AND ESTABLISHMENT OF Euseius stipulatus (Athias-Henriot) IN MIRAFLORES / LIMA / PERU}

\author{
Christian Enrique Valle Cabrejo ${ }^{1}$, Mónica Narrea Cango ${ }^{2}$ y Patricia Lizzet Llanos Chilet $^{3}$
}

\begin{abstract}
Resumen
Con el fin de determinar la fluctuación poblacional de Tetranychus sp. en la vegetación que cubre los acantilados del distrito de Miraflores en Lima, Perú y evaluar el efecto predador de Euseius stipulatus (Athias-Henriot), luego de un protocolo de liberación, se realizaron observaciones y muestreos en dos sectores de dicho acantilado: San Martín y Armendáriz. La evaluación se realizó entre octubre de 2013 y octubre de 2014, y consistió en dividir en cinco áreas cada sector y evaluar semanal y aleatoriamente, 20 hojas (haz y envés), para un total de 100 hojas por sector. De acuerdo a los resultados, la mayor infestación ocurrió en el sector San Martín, con una densidad de 74.9 individuos de Tetranychus sp./hoja, a un promedio de $24.1{ }^{\circ} \mathrm{C}$ y humedad relativa de $76.4 \%$; no hubo preferencia de la plaga por algún lado de la hoja. En el sector Armendáriz, la presencia de Tetranychus sp. fue menor, con una densidad de 11.2 individuos/hoja, a un promedio de $23.7^{\circ} \mathrm{C} \mathrm{y}$ humedad relativa de $76.3 \%$; se observó mayor población en el haz de las hojas. Las liberaciones realizadas de E. stipulatus sí influyeron en el control de Tetranychus sp.
\end{abstract}

Palabras clave: fluctuación, poblacional, Tetranychus, Euseius stipulatus, Ipomoea purpurea, Control Biológico.

\begin{abstract}
This research was conducted in two fields of Ipomoea purpurea (L.) Roth on the cliffs of Miraflores-Lima: "San Martín" and "Armendáriz", with the objectives to determine the population fluctuation of Tetranychus sp. and Euseius stipulates (Athias-Henriot) on the leaves of I. purpurea, and to determine the effect of the release of E. stipulatus into the Tetranychus sp. population. The evaluation lasted one year, from October 2013 to October 2014. The methodology consisted in dividing each sector into five areas and evaluating weekly and randomly, 20 leaves (upper and lower side), for a total of 100 leaves per sector. The higher infestation occurred in San Martin, with a density of 74.9 mites per leaf at $24.1{ }^{\circ} \mathrm{C}$ and $76.4 \%$ of relative humidity. In the Armendáriz sector, Tetranychus sp. presence was lower, with a density of 11.2 individuals / leaf at $23.7{ }^{\circ} \mathrm{C}$ and at $76.3 \%$ of relative humidity. In general, the higher peaks of Tetranychus sp. occur in summer when temperatures are higher and their cycles are shorter. Nevertheless, it was demonstrated that it has a damaging affect all year. Through the liberations, the predator action of E. stipulatus over the different stadiums of Tetranychus sp. was successfully demonstrated..
\end{abstract}

Key words: fluctuation, population, Tetranychus, Euseius stipulatus, Ipomoea purpurea, Biologic Control.

\section{Introducción}

Las plantas no solo nos ofrecen alimento, medicinas, madera, combustible y fibras, también producen el oxígeno que respiramos, mantienen el suelo, regulan la humedad y contribuyen a la estabilidad del clima, entre otros beneficios. A nivel urbano, y sobre todo en el área litoral, son muy importantes las plantas que cumplen funciones protectoras en la estabilización de los acantilados, pues las raíces de estas plantas se sujetan al sustrato y así previenen los desplazamientos de rocas; en los sectores más estables, la alfombra vegetal genera un perfil pedológico que funciona como una especie de «piel» protectora (FAO, 2014; CORIMAT, 2017).

En Lima/Perú, parte de la franja costera presenta numerosos acantilados cubiertos de cantos rodados susceptibles de desprenderse fácilmente y producir derrumbes hacia las partes bajas que contienen autopistas, playas o lugares de esparcimiento, por lo que han sido sembrados con plantas que mejoran su 
estabilidad, aumentando así la seguridad para dichos lugares. Específicamente en el distrito costero de Miraflores, la planta sembrada por excelencia, es la "campanilla", una planta rastrera cuyo desarrollo forma un manto de hojas verdes, que da mayor sentido al nombre de dicha zona, conocida coloquialmente como “Costa Verde" (Guzmán et al., 1997; Díaz, 2008). En los últimos años, esta importante cubierta vegetal ha sido atacada en forma estacional y por focos, por una especie de "arañita roja" (Tetranychus sp.) cuya población causa daños muy visibles y severos en toda la plantación.

$\mathrm{El}$ ataque estacional y no homogéneo de esta plaga podría explicarse según lo señalado por Cisneros et al., (1995), quienes indican que las poblaciones (de insectos), no mantienen una densidad constante sino que, con el transcurso del tiempo, presentan fluctuaciones más o menos marcadas donde se alternan altas y bajas densidades. Estas fluctuaciones suelen estar asociadas con las variaciones estacionales, con la acción de los enemigos naturales y con la relativa disponibilidad de alimento. Por su parte, Chant \& McMurtry (1994) indican que los fitoseidos son los más importantes predadores de tetraníquidos o arañas rojas.

Considerando lo antes mencionado, el presente trabajo de investigación tuvo como objetivo determinar la fluctuación poblacional del ácaro Tetranychus que ataca la "campanilla" de la franja costera del Distrito de Miraflores y evaluar si las liberaciones del ácaro predador E. stipulatus, tienen efecto controlador de esta plaga.

\section{Materiales y métodos}

El estudio se realizó de octubre de 2013 a octubre de 2014, en el ámbito de desarrollo de la "campanilla", que pertenece al acantilado miraflorino $\left(12^{\circ} 06^{\prime} 51^{\prime \prime} \mathrm{S}\right.$ $\left.77^{\circ} 03^{\prime} 27^{\prime \prime} \mathrm{O}\right)$. Este acantilado, que comprende aproximadamente $4.5 \mathrm{~km}$, se ubica al sur de la ciudad capital y sobre los $79 \mathrm{msnm}$; su cercanía al mar hace que el clima sea fresco pero casi siempre nublado, con una temperatura media anual de $18{ }^{\circ} \mathrm{C}$ y una humedad relativa que fluctúa entre $80 \%$ y $90 \%$. En dicho acantilado se identifican dos sectores: San Martín (3 $920 \mathrm{~m}^{2}$ ) y Armendáriz (9 $145.5 \mathrm{~m}^{2}$ ). San Martín es más cercano a la carretera por donde transita un alto número de vehículos, carece de árboles y por lo tanto es más expuesto a las corrientes de aire (Figura 1); Armendáriz presenta árboles y solo la parte baja es cercana a la carretera (Figura 2).

Fluctuación de Tetranychus sp.: Se realizaron evaluaciones semanales, cada sector en estudio, se dividió en 5 subsectores y dentro de ellos se eligieron 5 puntos al azar, colectándose 4 hojas por cada punto, para un total de 100 hojas por cada sector, durante cada una de las 51 evaluaciones realizadas. Al momento del muestreo, se contaron (con una lupa de 20X) huevos e individuos de Tetranychus sp. y ácaros predadores liberados (individuos), tanto en el haz como en el envés, reportándose el número promedio de ambos por hoja. En el caso de la plaga, el número también se reportó en Grados (1 al 6) según la escala de Sánchez \& Sarmiento (2012): Grado 1 = 0; Grado $2=1$ a 5; Grado $3=6$ a 10; Grado $4=11$ a 24; Grado $5=26$ a 50, y Grado $6=$ más de 50 individuos.

Para determinar el tipo de distribución de Tetranychus sp. en la "campanilla", se aplicó la ecuación propuesta por Ramírez (1999) (Ecuación 1):

$$
\mathrm{DP}=\sigma^{2} / \mu \ldots \text { Ecuación } 1 .
$$

Donde:

DP = Distribución Población;

$\sigma^{2}=$ Varianza poblacional; $y$

$\mu=$ Media muestral.

Si la $\mathrm{DP}=>1$, la distribución es agregada;

$\mathrm{Si}$ la $\mathrm{DP}=1$, es al azar; y si la $\mathrm{DP}=<1$, es uniforme.

Se entiende que cuando la población exhibe heterogeneidad en sus densidades la varianza será mayor y, por lo tanto, corresponderá a una distribución tipo agregada. Si hay una baja variabilidad entre las muestras, el patrón de distribución será uniforme.

Liberaciones del predador E. stipulatus: Con un pie de cría, proveniente del SENASA (Servicio Nacional de Sanidad Agraria), se realizó la crianza masiva de $E$. stipulatus en hojas de "acalifa" (Acalypha wilkesiana), de aproximadamente $70 \mathrm{~cm}^{2}$ de área. Cada hoja, o unidad de crianza, se cubrió en sus bordes con tiras de papel toalla para evitar la fuga de los ácaros y se colocó sobre esponjas embebidas en agua; como alimento se les ofreció polen de "higuerilla" (Ricinus communis). Para conocer la producción de cada unidad de crianza, se contabilizó la población generada de predadores, de 20 hembras y 10 machos, en un lapso de 20 días.

De acuerdo al protocolo establecido, las liberaciones se realizaron luego de observar un aumento de la plaga. Estas liberaciones fueron de tres tipos (Tabla 1).

Tabla 1. Liberaciones de Euseius stipulatus, realizadas para el control de Tetranychus sp. en el acantilado de Miraflores, Perú.

\begin{tabular}{ll}
\hline Tipo & Detalle \\
\hline L-1 & $\begin{array}{l}\text { Con un pincel se contó y trasladó a los predadores } \\
\text { sobre una nueva hoja dentro de un vaso plástico } \\
\text { de } 250 \text { ml, tapado con papel toalla. En campo, se } \\
\text { abrieron los vasos y se esparcieron los individuos }\end{array}$ \\
& $\begin{array}{l}\text { sobre el follaje (Figura 3a). } \\
\text { L-2 }\end{array}$ \\
& $\begin{array}{l}\text { Cada unidad de crianza se colocó en tapers de 11, } \\
\text { tapadas con papel toalla. En campo, se abrieron }\end{array}$ \\
& $\begin{array}{l}\text { los tapers y se dejó la hoja sobre el follaje, } \\
\text { L-3 }\end{array}$ \\
& $\begin{array}{l}\text { Con un aspirador manual, se contó y retiró los } \\
\text { predadores dentro de frascos plásticos con } \\
\text { aserrín, cerrando la entrada con la tapa. En campo, }\end{array}$ \\
& se abrieron los frascos y se vaciaron sobre el \\
& follaje (Figura 3c).
\end{tabular}




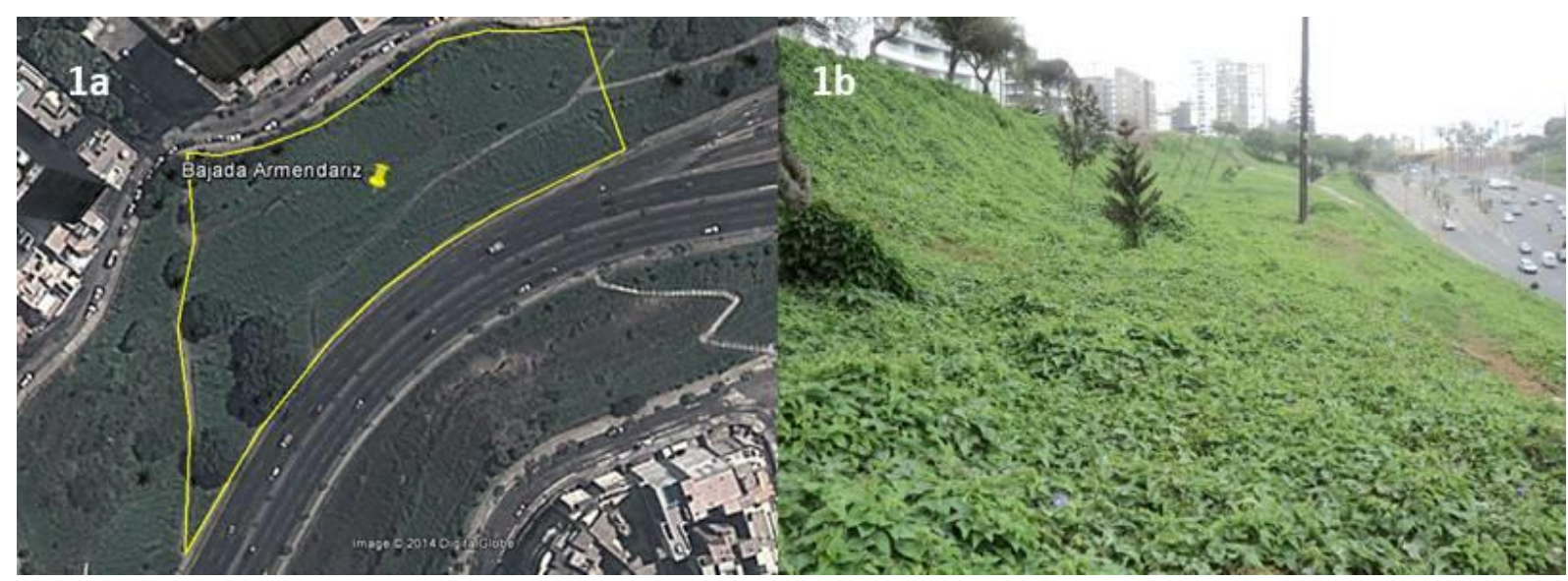

Figura 1. Sector San Martín a) Mapa del sector. b) Detalle del sector. Miraflores, Lima-Perú. Foto: Valle, 2013.

En cuanto a las condiciones ambientales, los datos de temperatura $\left(\mathrm{t}^{\circ}\right)$ y humedad relativa $(\mathrm{hr})$, fueron proporcionados por el Servicio Nacional de Meteorología e Hidrología del Perú - SENAMHI.

\section{Resultados y discusión}

Usando claves taxonómicas de la North American Plant Protection Organization (NAPPO) del 2014, se identificó la plaga como Tetranychus sp. (Acari: Tetranychidae). No se llegó al nivel específico, por no encontrar en dichas claves las características morfológicas que coincidieran con la especie que ataca a la "campanilla". La identificación como tal, fue confirmada por el Laboratorio de Entomología del SENASA. En cuanto a la "campanilla", de acuerdo a las claves de Alarcón-Bravo et al. (2016), se determinó que corresponde a Ipomoea purpurea (L.) Roth. Brako \& Zarucchi (1993), la reportan entre los 1000 a 2000 msnm; pero en Chiclayo-Perú, Alarcón-Bravo et al. (2016) la reportan entre los 18 a $1554 \mathrm{msnm}$.

\section{Fluctuación del ácaro plaga}

a.- Fluctuación en el Sector San Martín (Figuras 4 y 5).

En este sector, al inicio del estudio y hasta noviembre del 2013, la población de individuos y huevos de Tetranychus sp. fue muy baja, en promedio menos de 1/hoja (Grado 1); esto se dio bajo condiciones promedio de $18.1^{\circ} \mathrm{C}$ y $79.8 \% \mathrm{hr}$. Sin embargo, a partir de diciembre de 2013, empezó a incrementarse, tanto el número de individuos como de posturas.

Posteriormente, entre el 19 de diciembre del 2013 y el 16 de febrero del 2014, la población de Tetranychus sp. se incrementó drásticamente, reportándose más de 50 individuos (Grado 6) y 107.8 huevos por hoja, respectivamente; este aumento de la población ocurrió en el periodo de más alta temperatura y baja humedad relativa $\left(24.1{ }^{\circ} \mathrm{C}\right.$ y $\left.76.4 \% \mathrm{hr}\right)$ y coincide con lo reportado por Reyes et al. (2011), en Colombia, quienes encontraron altas poblaciones del ácaro Oligonychus yothersi (McGregor) cuando, en su estudio registraron altas temperaturas y baja humedad relativa $\left(26 \pm 3{ }^{\circ} \mathrm{C} 56 \pm 3 \% \mathrm{hr}\right)$.

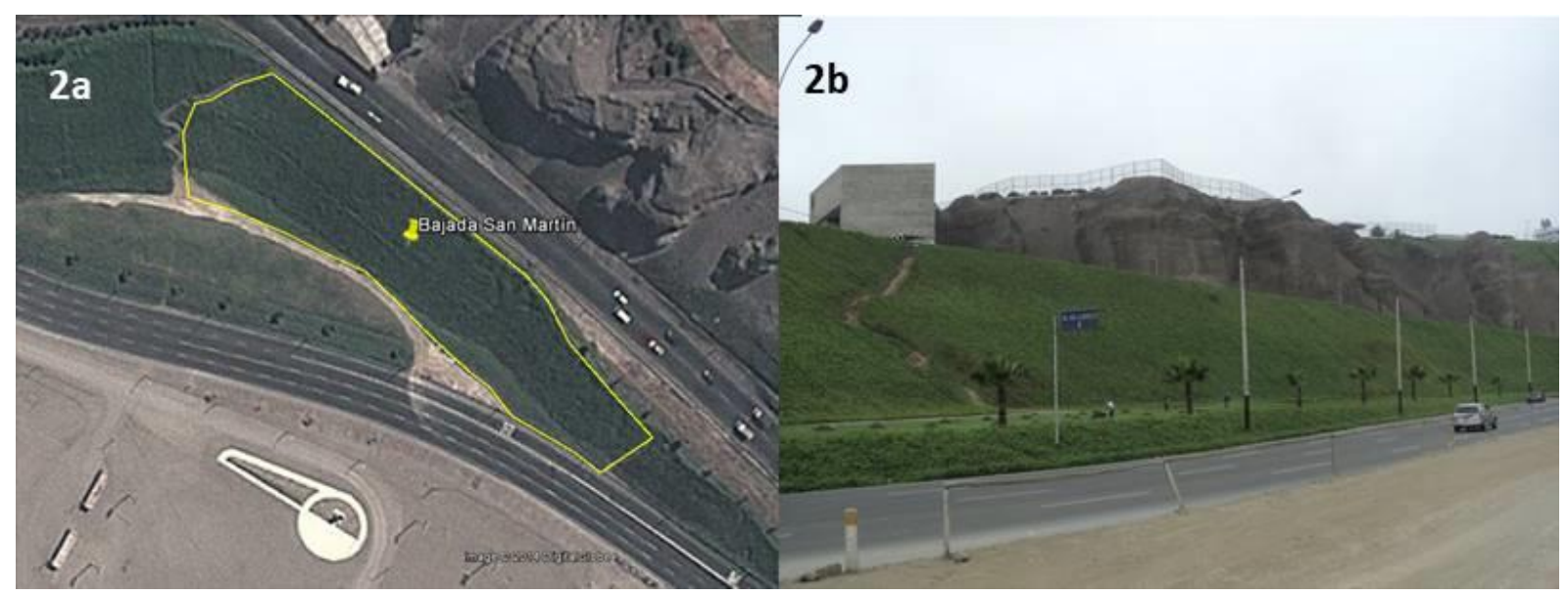

Figura 2. Sector Armendáriz a) Mapa del sector. b) Detalle del sector. Miraflores, Lima - Perú. Foto: Valle, 2013. 


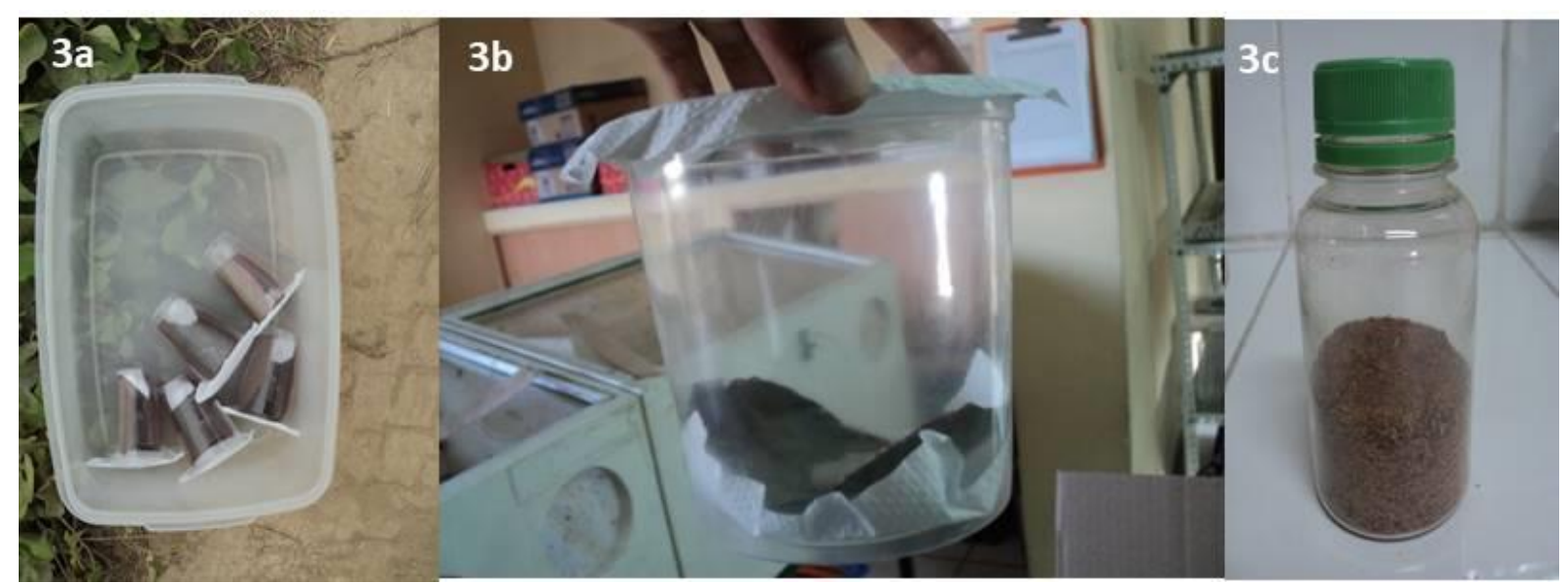

Figura 3. Tipo de Liberaciones a) Tipo L-1. b) Tipo L-2. c) Tipo L-3. Miraflores, Lima-Perú. Foto: Valle, 2013.

Conforme al protocolo, cuando se observó el incremento de la población de Tetranychus sp., se realizaron las primeras liberaciones de E. stipulatus (Figura 3). Las liberaciones tipo L-1 se hicieron en tres oportunidades los días 17, 19 y 30 de diciembre, con aproximadamente 500 individuos cada una. Este tipo de liberación no tuvo el efecto esperado presumiblemente por el bajo número de predadores liberados, debido a que Tetranychus sp. siguió incrementando su población, lo que demostró el nulo control que se ejerció; sin embargo, coincidentemente, también el día 16 de enero se registró el pico poblacional de $E$. stipulatus, con 1.9 ácaros/hoja, lo que evidencia la influencia del crecimiento de la población de la plaga sobre la del predador, confirmando lo expuesto por Badii et al. (2010), quienes señalaron que los cambios poblacionales de los fitoseidos están asociados íntimamente con el patrón de fluctuación poblacional de los tetraníquidos.

La alta población de Tetranychus sp. provocó daños severos a la "campañilla", por lo que, ante la falta de control del predador, fue necesario aplicar un agroquímico, seleccionándose D-Octil (Sulfosuccinato sódico al 70\%), cuyo uso se recomienda en forma de lavados para el control de ácaros Tetranychus. Debido a que su efecto no fue inmediato, tuvieron que hacerse hasta cuatro lavados, entre el 07 y el 29 de enero, para bajar la población Tetranychus sp. a Grado 3.

Complementando el manejo de la plaga, el 06 de febrero nuevamente se iniciaron las liberaciones del acaro predador, esta vez del tipo L-2, con 7480 individuos. Posteriores liberaciones fueron del tipo L-3 y se realizaron los días 13 y 27 de marzo, 19 y 20 de junio, 01 de julio y 15 de julio, para un total de 12352 predadores. De acuerdo a los resultados, estas liberaciones sí contribuyeron al control de la plaga, pues la misma comenzó a descender progresivamente al punto que del 13 de marzo al 03 de octubre, los registros indicaron que la población de Tetranychus sp., bajó y se mantuvo en Grado 1 y las posturas en menos de 1 huevo/hoja; sólo el día 26 de junio los individuos

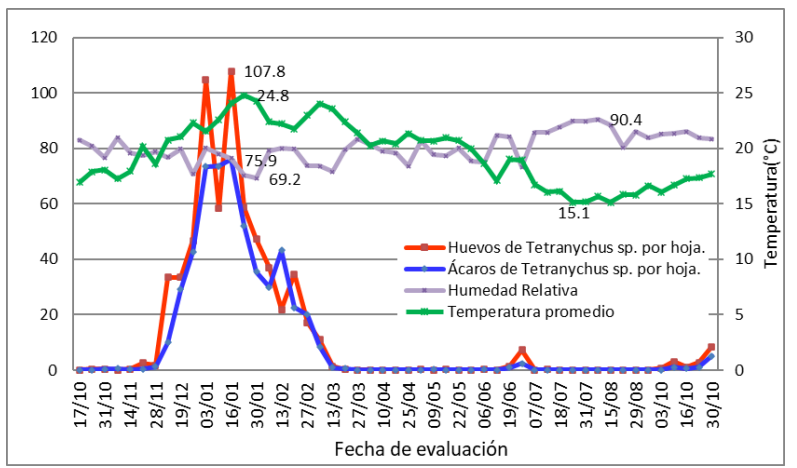

Figura 4. Fluctuación poblacional en el sector San Martín de Tetranychus sp. en Ipomoea purpurea. Miraflores, Lima - Perú.

llegaron a Grado 2. Cabe indicar que a pesar de que en varios de los muestreos no se encontró ningún individuo o huevo de Tetranychus sp., casi siempre se encontraron individuos de E. stipulatus, aunque en bajas densidades; esto da evidencia de su carácter generalista ya determinado por Ferragut \& Escudero (1997), que le permite subsistir con diferentes ofertas de comida (otros ácaros y polen, presentes en la Ipomoea purpurea). Considerando que I. purpurea es una planta perenne, la existencia de predadores durante todo el año puede generar una respuesta más rápida cuando Tetranychus sp. eleva su población principalmente en verano.

A parte del factor predador, otros factores a considerar que explicaran el descenso de la plaga fueron las condiciones ambientales adversas (descenso de la temperatura hasta $14.0{ }^{\circ} \mathrm{C}$ e incremento de hasta $90.4 \%$ de hr); ambos factores, predador y condiciones ambientales, causaron en forma conjunta un mejor efecto.

Entre el 10 y el 30 de octubre, lapso en el cual la temperatura empezó a incrementarse (hasta llegar a un promedio de $19.6{ }^{\circ} \mathrm{C}$.), Tetranychus sp. también fue incrementando su población hasta llegar en la última evaluación a 5.0 ácaros/hoja (Grado 2), y 8.5 
huevos/hoja, dando inicio a una nueva etapa fenológica.

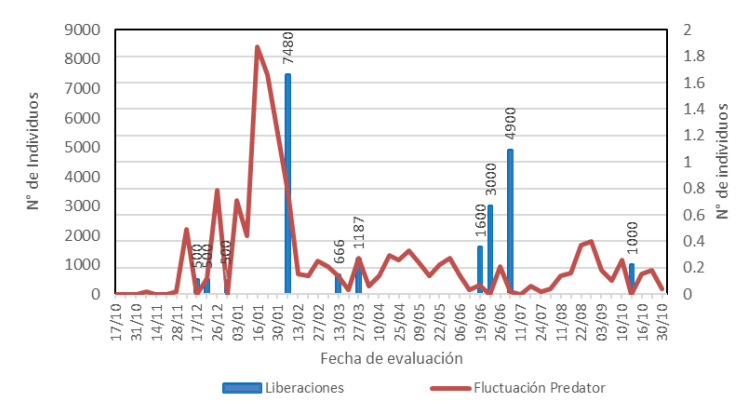

Figura 5. Fluctuación poblacional en el sector Armendáriz de Tetranychus sp. en Ipomoea purpurea. Miraflores, Lima - Perú.

En cuanto a la distribución de Tetranychus sp. en este sector, se encontró que en el $97.4 \%$ de las evaluaciones en las que estuvo presente, estuvo dispersa en forma agregada y, en el $2.6 \%$ restante, tuvo una distribución aleatoria. Esta característica coincide con Chacón-Hernández et al. (2016), en un estudio sobre $T$. urticae, quienes encontraron que, en todos los muestreos, esta especie mostró una distribución agregada.

b.- Fluctuación en el Sector Armendáriz (Figuras 6 y 7).

En este sector, Tetranychus sp. no alcanzó grandes poblaciones; máximo llegó a Grado 4. Al inicio de la evaluación, e igual que en el sector San Martín, la población de individuos y huevos fue muy baja, registrándose en muchas de las evaluaciones cero individuos. Sólo entre el 09 y el 24 de enero la población se incrementó hasta Grado 4, con picos poblacionales de 11.2 ácaros/hoja y 19.8 huevos/hoja, los días 09 y 17 de enero, respectivamente. Estos valores se alcanzaron cuando la temperatura registró uno de sus más altos valores $\left(24.2^{\circ} \mathrm{C}\right)$ y la hr fue muy baja $(76.3 \%)$. En vista que el desarrollo de la plaga causó serios daños a I. purpurea, se procedió a realizar liberaciones de E. stipulatus, tipo L-2, los días 10, 21 y 29 de enero, para un total de 19472 individuos, lo que causó un control efectivo, ya que posteriormente la población de Tetranychus sp. descendió hasta Grado 1 y eventualmente Grado 2. Las últimas liberaciones, que fueron del tipo L-3, se realizaron cuando las evaluaciones señalaban el incremento de la plaga a Grado 2; es así que entre el 28 de marzo y el 27 de junio, se liberaron un total de 16920 individuos, distribuidos en 11 ocasiones. Al igual que en el sector San Martín, en general se observó que, independientemente de las liberaciones, hubo una relación directa entre la presencia de la plaga y el de la población de $E$. stipulatus. En este sector no se realizó ninguna aplicación de productos químicos.
En cuanto a la distribución de la población, del total de evaluaciones en las que se evidenció la plaga, en el $89.4 \%$ se reportó una distribución agregada, en el $6.4 \%$ fue aleatorio y en el $4.3 \%$, uniforme. La predominante distribución agregada, tanto en este sector como en San Martín, puede atribuirse a que estos ácaros viven y se desarrollan en colonias (Ramos, 2012) y, por lo tanto, su desarrollo se da en forma aislada por grupos; las demás distribuciones espaciales solo se observan a densidades muy pequeñas al inicio de las infestaciones, cuando las hembras recién están empezando a colonizar las hojas.

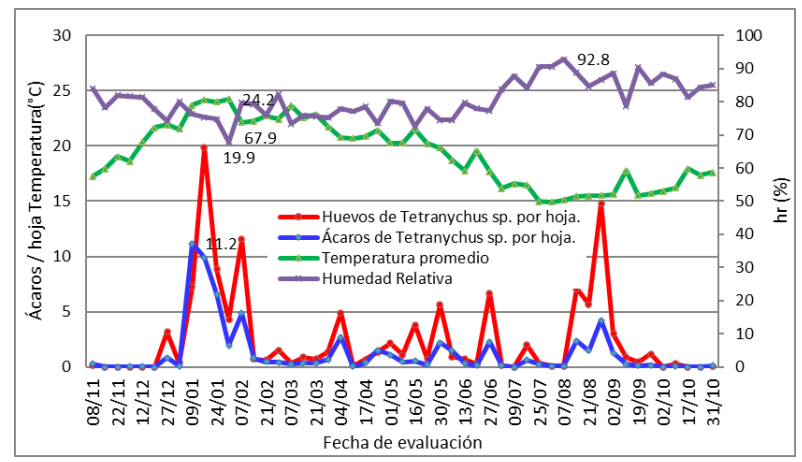

Figura 6. Fluctuación poblacional en el sector San Martín de Euseius stipulatus en Ipomoea purpurea. Miraflores, Lima-Perú.

En cuanto a la diferencia que presenta el desarrollo de la plaga entre ambos sectores evaluados, se debe a la cercanía a la carretera y la presencia/ausencia de árboles, que hacen que el sector San Martín sea el más afectado por las corrientes de aire y la presencia de polvo por el tránsito vehicular, factores que en definitiva favorecen la presencia y capacidad distributiva de Tetranychus sp.; además, se observó un factor adicional, el del estrés hídrico que soporta el Sector San Martín, por lo difícil de su acceso, que dificulta la llegada del personal responsable del riego. Este último factor llegó a ser especialmente intenso durante los meses de verano, cuando se observó precisamente mayor daño de la plaga.

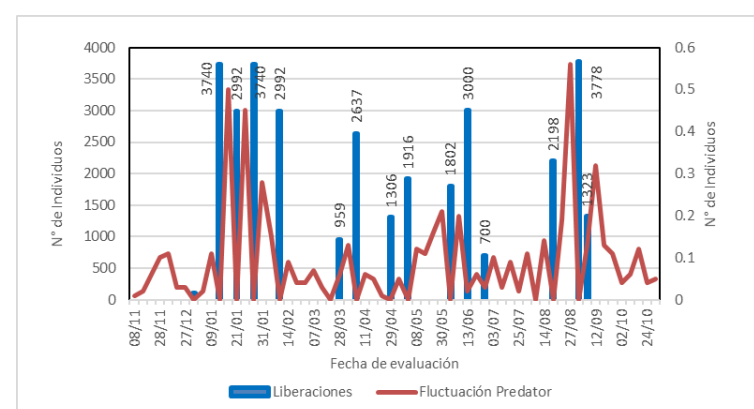

Figura 7. Fluctuación poblacional en el sector Armendáriz de Euseius stipulatus en Ipomoea purpurea. Miraflores, Lima-Perú. 


\section{Preferencia posicional}

En el caso del sector San Martín, los individuos de Tetranychus sp. fueron encontrados de manera equitativa entre ambos lados, demostrando no tener preferencia. En el caso de la oviposición, se observó preferencia por hacerlo en el haz $(64.6 \%)$ y en el caso de E. stipulatus, al igual que su alimento, significativamente no tuvo preferencia por algún lado de la hoja. En el caso del sector Armendáriz, tanto los individuos como los huevos de Tetranychus sp., fueron encontrados preferentemente en el haz, siendo que en el caso de los huevos se encontró una mayor prevalencia $(91.9 \%)$. Con relación al predador, al igual que en lo ocurrido en San Martín, no hubo preferencia por alguno de los lados de la hoja, aunque se encontró mayor porcentaje en el envés (55.4\%).

Los resultados similares entre ambos sectores, no demuestran que las Tetranychus sp., o su predador, tuvieran preferencia por algún lado de la hoja, lo que contradice lo reportado por Ramos (2012), quien señala que los fitoseidos presentan acarodomatia, o preferencia por el envés de las hojas, donde los pelos finos y nervaduras les otorgan refugio y protección contra la desecación producida por la luz solar y las corrientes de aire.

Efecto del tipo de liberaciones

Los métodos de liberación L2 y L3 fueron los más efectivas en cuanto a su preparación y volumen de liberación, y no presentaron dificultades en su ejecución. En cuanto al método L-1, no se consideró efectivo y se desestimó, por la complejidad de su preparación, que demanda mucho tiempo y solo permite liberar poblaciones pequeñas.

\section{Agradecimientos}

A la empresa Caña Brava por el financiamiento y por facilitar los materiales e instalaciones de su laboratorio y en especial a la Ing. Gaby Cárdenas por su apoyo durante el desarrollo de la investigación.

\section{Literatura citada}

Alarcón-Bravo L., Torres-Reaño G., Austin D., Rojas-Idrogo C. \& Delgado-Paredes G. 2016. Sinopsis de Ipomoea L. y Merremia Dennst. Ex Endl. (Convolvulaceae) en el norte del Perú (Lambayeque y territorios adyacentes). Acta Botánica Malacitana, 41(1): 101-120. Málaga, España.

Badii M., Landeros J. \& Cerna E. 2010. Regulación Poblacional de Ácaros Plaga de Impacto Agrícola. Daena: International Journal of Good Conscience, 5(1): 270-302.

Brako L. \& Zarucchi J. 1993. Catalogueof the flowering plants and gymnosperms of Perú / Catálogo de las Angiospermas y Gimnospermas del Perú. Monographs in Systematic Botany from the Missouri Botanical Garden, 45: 365-374.
Chacón-Hernández J., Ruiz-Díaz A., Cerna-Chávez E., Ochoa-Fuentes Y., Hernández-Juárez A., Torres-Castillo J. \& Landeros-Flores J. 2016. Efectos de Phytoseiulus persimilis (Athias-Henriot) sobre tablas de vida de Tetranychus urticae Koch en cuatro variedades de rosa. Southwestern Entomologist, 41: 567-576.

Chant D. \& McMurtry J. 1994. A. review of the subfamilies Phytoseiinae and Typhlodrominae (Acari: Phtyoseiidae). Internat. J. Acarol., 20: 223-310.

Cisneros F., Alcázar J., Palacios M. \& Ortiz O. 1995. Una estrategia para el desarrollo e implementación del Manejo integrado de plagas. CIP-Circular, 21(3): 2-7. Centro Internacional de la Papa. Lima, Perú.

CORIMAT, 2017. Red Atlántica para la Gestión de los Riesgos Costeros-- Soluciones alternativas para la protección de las costas. Director de la publicación: Alain Rousset. Edición BRGM, ONF (Francia).

Díaz J. 2008. Estabilización del Talud de la Costa Verde en la Zona del Distrito de Magdalena. Tesis para optar por el título de Ingeniero Civil. Pontificia Universidad Católica del Perú Facultad de Ciencias e Ingeniería.

FAO, 2014. Organización de las Naciones Unidas para la Alimentación y la Agricultura. Una huerta para todos Manual de auto-instrucción. 5ta Edición revisada y ampliada Chile.

Ferragut F. \& Escudero A. 1997. Taxonomía y distribución de los ácaros depredadores del género Euseius Wainstein 1962, en España (Acari: Phytoseiidae). Boletín de Sanidad Vegetal, Plagas, 23: 227-235.

Guzmán A., Zavala B., \& Valenzuela G. 1997. Estudio de la seguridad física de los acantilados de la Costa Verde. Boletín $\mathrm{N}^{\circ} 18$, Serie C: Geodinámica e Ingeniería Geológica. Instituto Geológico Minero y Metalúrgico (INGEMMET)/MINEM/PERÚ.

URI: http://repositorio.ingemmet.gob.pe/handle/ingemmet/24 0 .

McDonald A. 1994. Convolvulaceae (II). En: Sosa, V. (ed.). Flora de Veracruz. Fascículo 77. Instituto de Ecología. Xalapa, Veracruz, México.

NAPPO (North American Plant Protection Organization). 2014. PROTOCOLOS DE DIAGNÓSTICO -PD 03: Identificación morfológica de las arañas rojas (Tetranychidae) que afectan a las frutas importadas. Secretaría de la Organización Norteamericana de Protección a las Plantas 1431 Merivale Road, 3rd Floor, Room 140. Ottawa, Ontario, Canadá.

Ramírez G. 1999. Ecología Aplicada. 1ed., Fundación Universidad de Bogotá Jorge Tadeo Lozano. Bogotá, Colombia.

Ramos M. 2012. Phytoseiidae. En: Estrada E, Acuña J, Martha C. \& Equihua A. (eds.). Ácaros de importancia agrícola. Texcoco, MX, Colegio de Postgraduados. 223249.

Reyes J., Mesa N. \& Kondo T. 2011. Biología de Oligonychus yothersi (Mcgregor) (Acari: Tetranychidae) sobre aguacate Persea americana Mill. cv. Lorena (Lauraceae). Caldasia, 33(1): 201-220. Instituto de Ciencias Naturales / Facultad de Ciencias / Universidad Nacional de Colombia. Bogotá/Colombia. URL: https://revistas.unal.edu.co/index.php/cal/article/view/36 387.

Sánchez G. \& Sarmiento J. 2012. Evaluación de insectos. 5 ed., Universidad Nacional Agraria La Molina. Departamento de entomología. Lima Perú. 
${ }^{1}$ Universidad Nacional Agraria La Molina \Facultad de Ciencias. Apartado Postal 12-056. Lima 12 - Perú.

${ }^{2}$ Universidad Nacional Agraria La Molina \Facultad de Agronomía \Departamento de Entomología. Apartado Postal 12-056. Lima 12 - Perú. Autor de correspondencia: mnarrea@lamolina.edu.pe.

${ }^{3}$ Municipalidad de Miraflores $\backslash$ Sub gerencia de limpieza pública y áreas verdes. Av. Larco N400, Miraflores / Lima / Perú. 